\title{
The Effects of Dynamic Loading on Hysteretic Behavior of Frictional Dampers
}

\author{
Hamid Rahmani Samani, ${ }^{1}$ Masoud Mirtaheri, ${ }^{1}$ Amir Peyman Zandi, ${ }^{1}$ and Hamid Bahai ${ }^{2}$ \\ ${ }^{1}$ Department of Civil Engineering, K.N. Toosi University of Technology, P.O. Box 15875-4416, Tehran 19697 64499, Iran \\ ${ }^{2}$ School of Engineering \& Design, Brunel University, Uxbridge, London UB8 3PH, UK \\ Correspondence should be addressed to Hamid Bahai; hamid.bahai@brunel.ac.uk
}

Received 26 January 2014; Accepted 14 May 2014; Published 3 June 2014

Academic Editor: Gyuhae Park

Copyright (C) 2014 Hamid Rahmani Samani et al. This is an open access article distributed under the Creative Commons Attribution License, which permits unrestricted use, distribution, and reproduction in any medium, provided the original work is properly cited.

\begin{abstract}
During an earthquake excitation, a frictional damper may experience many cycles of dynamic loading. The effects of wear and heat induced by the cyclic loading result in the possible decay of the slippage load which subsequently reduce the energy absorption of the damper. In this paper, the effect of dynamic loading on hysteretic behavior of a special kind of frictional damper, namely, cylindrical frictional damper (CFD), is investigated by experimental means as well as numerical models which also account for coupled thermal-structural interaction. The damper is deemed to be more susceptible to thermal deformations due to the shrinkfit mechanism by which the device is assembled. The numerical models are validated experimentally and may be utilized for simulation of dynamic cyclic loading on frictional dampers. The results demonstrate that the slippage load is reduced gradually when subjected to consecutive cycles. This drift is attributed to thermal deformation. The verified numerical models are used to improve the geometry of the CFD. With the geometrical improvements implemented, the subsequent numerical studies confirmed that almost no degradation of the slippage load occurred. Furthermore, a dimensionless parameter is introduced by the authors which shows the effect of wear on the response of CFDs.
\end{abstract}

\section{Introduction}

Frictional devices have long been used in mechanical and structural engineering for absorbing and dissipating energies. Efficiency and convenience of such dampers make them appropriate candidates for damping mechanism under dynamic loadings. Utilizing frictional based dampers in earthquake resistant structures has many advantages over the other types of energy dissipating devices. These include low cost of manufacturing and maintenance and also being less susceptible to environmental effects, as well as being less sensitive to the change of ambient temperature. Furthermore, no yielding occurs in such dampers after a severe earthquake which eliminates the need for replacement.

Pall and Marsh [1] first proposed sliding plate frictional dampers to be installed at the crossing joint of the Xbraces. Various other types of frictional devices have been proposed by other researchers. Wu et al. [2] introduced the improved Pall frictional damper (IPFD), which replicates the mechanical properties of the Pall frictional damper but offers some advantages in terms of ease of manufacture and assembly. The Sumitomo friction damper [3] utilizes a more complicated design when compared to the Pall frictional damper. The precompressed internal spring exerts a force that is converted through the action of inner and outer wedges into a normal force on the friction pads. Fluor Daniel Inc. developed and tested another type of frictional device which is called energy dissipating restraint (EDR) [4]. The design of this friction damper is similar to the Sumitomo friction damper, since this device also includes an internal spring and wedges encased in a steel cylinder. The EDR utilizes steel and bronze friction wedges to convert the axial spring force into normal pressure on the cylinder. Mualla and Belev [5] proposed a friction damping device (FDD) and carried out tests for assessing the friction pad material. Monir and Zeynali [6] introduced and tested a modified 
friction damper (MFD) which is similar to the Pall friction damper; however it is applied in the diagonal bracing. Most of the above frictional dampers are made of a set of metal plates which relies on coefficient of friction and clamping force provided by bolting system. The relaxation or loosening of the clamping elements such as spring or bolts through time may lead to decay of slippage load. Chen et al. [7] introduced piezoelectric friction damper (PFD). The clamping force in such a damper is regulated by piezoelectric actuators. However, piezoelectric based frictional dampers are not applicable to building structures due to the fact that the force produced by a piezoelectric actuator is rather small in value. Moreover piezoelectric actuators are not cost-effective. Agrawal and Yang [8] proposed an electromagnetic frictional damper. This device is based on the regulation of friction force across the damper using electromagnetic field. Similar to piezoelectric ones, activating force of electromagnetic based devices can also produce forces which are rather small in value. Recently, Mirtaheri et al. [9] proposed an innovative type of frictional damper called cylindrical frictional damper (CFD). CFDs which are made of stainless steel produce friction through surfaces without using any type of clamping elements such as springs or bolts. In addition construction cost is reduced due to the simplicity of the system. These dampers consist of two main parts, the inner shaft and the outer cylinder. These two parts are assembled such that one is shrink-fitted inside the other.

During an earthquake excitation, a frictional damper may experience many cycles of dynamic loading. The effect of wear and heat induced by the cyclic loading results in the possible decay of the slippage load. This is regarded as a major concern. Therefore, according to various specifications such as ASCE/SEI 41-06 [10] any type and size of the dampers used in the design must be tested through construction of prototypes before utilizing them in a real structure. Such prototypes must be tested with twenty fully reversed cycles at a frequency equal to the fundamental frequency of the structure. Performing such dynamic cyclic tests for dampers with large slippage loads and large stroke lengths require actuators that are very large in size and making them impractical to use. Therefore, the use of verified numerical models is a good substitute for experimental tests required by specifications.

The goal of this paper is to investigate the effects of dynamic loading on the hysteretic behavior of CFDs and the heat generated by the friction, which in turn may lead to the decay of energy absorption capacity of the system. Thermal effects due to friction are also valid for other types of dampers such as viscous or viscoelastic ones. However, CFD is more susceptible to this problem due to fact that the clamping force of such damper is provided by shrink-fit mechanism. The effect of wear induced by dynamic loading is also investigated.

Dynamic loading tests are performed at two different frequencies of $0.45 \mathrm{~Hz}$ and $0.7 \mathrm{~Hz}$ which are close to typical midrise buildings frequencies. Numerical models are also developed to investigate the hysteretic behavior of CFD by a series of coupled thermal-structural analyses. These models are compared and verified by the experimental results. After the verification of the models they are used for optimization of geometry of the damper parts for more stable hysteretic behavior. This is done through some simple but important changes in the geometry of CFD which lead to nearly negligible amount of decay in the hysteretic behavior of this damper.

\section{The Effects of Heat and Wear on Hysteretic Behavior of Frictional Dampers}

The movement of the frictional damper parts creates heat which leads to rise in the temperature. This heat is then transferred through elements within the damper. The increase of the temperature may influence the damper behavior in two ways, one is thermal deformation of the elements and the other is changing the mechanical and finishing properties of the sliding surfaces which subsequently influence the rate of wear.

On the other hand, wear itself can affect the hysteretic behavior of the damper. From an engineering point of view, wear is often classified as either mild with a low wear rate or severe with a high wear rate [11]. The former is a process that makes contact surfaces smoother than the original state, while the latter makes contact surfaces rougher than the original state. Therefore, wear may affect the hysteretic behavior of the damper in two ways; one is removal of material from contact surfaces which results in thinner plates and less clamping force and the other is changing the coefficient of friction.

Therefore, when dynamic loading is considered in frictional dampers, structural and thermal processes are much interconnected. These processes may lead to increase or decrease of slippage load over the cycles. Thus, in order to have a stable hysteretic behavior, one should investigate the governing factors. Accordingly, appropriate geometric parameters can be selected for the design of damper elements. In this study, taking advantage of analytical and experimental methods, governing parameters are calibrated such that a stable hysteretic behavior is achieved.

\section{Cylindrical Frictional Damper}

CFD was proposed by Mirtaheri et al. [9] as an innovating type of frictional damper which does not use bolts or any other mechanical pretension element to induce friction between contact surfaces. The main parts of CFD are the shaft and the cylinder as shown in Figure 1. These two parts are assembled such that one is shrink-fitted inside the other. The inner diameter of cylindrical element is slightly smaller than the diameter of the shaft at the contact length, $L_{0}$. Heating the cylindrical part leads to an increase of its diameter and the unheated shaft can be easily placed into the cylinder. The clamping pressure will be developed between the contact surfaces when thermal equilibrium is reached. If the damper force exceeds the static friction load, the shaft will move inside the cylinder and results in considerable mechanical energy absorption by friction. 


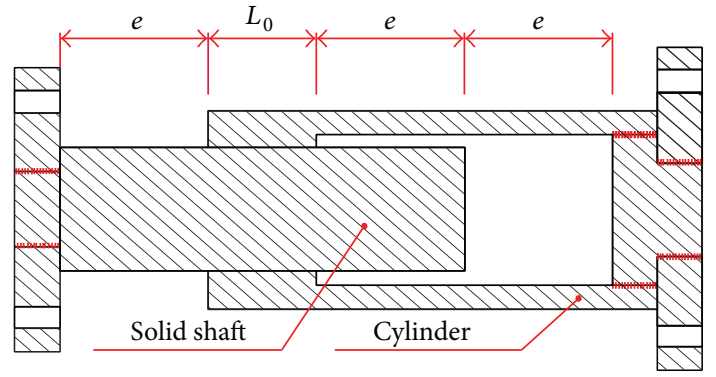

(a)

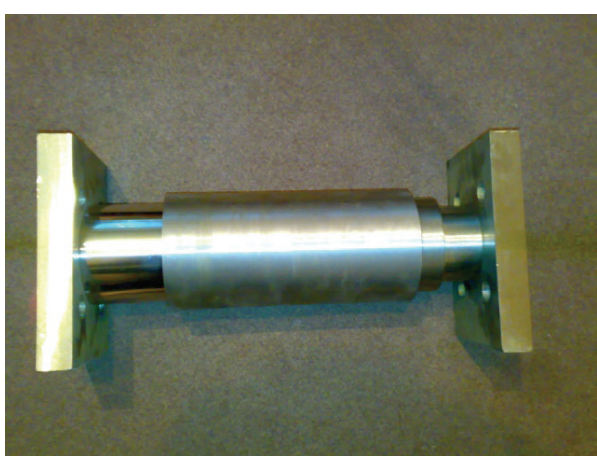

(b)

FIgUrE 1: The CFD: (a) longitudinal section of CFD; (b) assembled CDF.

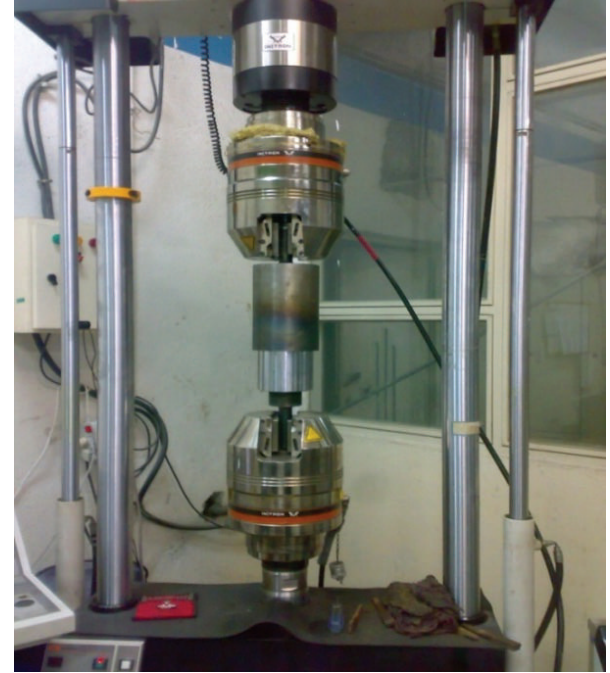

FIGURE 2: Test setup.

\section{Experimental Study of CFD under Dynamic Loading}

4.1. CFD Prototype Fabrication. A $200 \mathrm{kN}$ capacity CFD specimen made of steel with chrome coated shaft and highly polished contact surface of the cylinder is used for this experiment. This capacity is a typical value obtained by analysis of medium rise buildings. The coefficients of static and dynamic frictions $\left(\mu_{s}, \mu_{k}\right)$ are obtained based on several tests. The values of $\mu_{s}$ and $\mu_{k}$ are found to be 0.286 and 0.224 , respectively. Special techniques are utilized to improve contact surfaces in order to make $\mu_{s}$ and $\mu_{k}$ closer to each other.

4.2. Test Setup. CFD specimen is tested in vertical position as shown in Figure 2 by a universal testing machine. The actuator has a capacity of $250 \mathrm{kN}$. Figure 3 shows the connection of CFD specimen to the actuator. The actuator is equipped with an internal linear variable displacement transducer (LVDT) and a load cell in line with the cylinder, with both connected to an electronic data acquisition system.
TABLE 1: Specification of test cases.

\begin{tabular}{lccc}
\hline Test Case & Number of cycles & Stroke & $\begin{array}{c}\text { Frequency of } \\
\text { loading }(\mathrm{Hz})\end{array}$ \\
\hline Case A & 7 & $\pm 50 \mathrm{~mm}$ & 0.45 \\
Case B & 7 & $\pm 50 \mathrm{~mm}$ & 0.45 \\
Case C & 10 & $\pm 40 \mathrm{~mm}$ & 0.7 \\
\hline
\end{tabular}

4.3. Experimental Results. The hysteretic behavior of the original CFD is achieved under two different frequencies of $0.45 \mathrm{~Hz}$ and $0.7 \mathrm{~Hz}$. Tests are carried out at displacement levels of $40 \mathrm{~mm}$ and $50 \mathrm{~mm}$ which are below the level of the lockout of the damper. The test cycles and rates, at which the tests were performed, are shown in Table 1. After each test case the specimen is cooled down before performing the next test.

Figures 4, 5, and 6 represent hysteretic force-displacement curves of the test cases $\mathrm{A}$ to $\mathrm{C}$, respectively. As it was shown in previous research [9], the CFD exhibits classical rectangular hysteresis loops of frictional dampers. Almost the same performance is recorded in compression and tension. Table 2 compares the slippage load of each cycle of loading with the average value for test cases $\mathrm{A}$ to $\mathrm{C}$. The maximum deviations from average slippage loads are 14.82, 15.66, and 15.12 for test cases A, B, and C, respectively. As it can be noticed, the slippage load of the prototype is clearly drifted down in consecutive cycles. The drift in the slippage load may be considered as the result of wear in contact surfaces. However, comparing the slippage load of test cases A and B, in which all test conditions are similar, reveals the fact that the drift is due to effect of heat which is generated in the internal elements of the damper during the tests. In test case A, seven cycles of loading are applied to the specimen at frequency of $0.45 \mathrm{~Hz}$. Subsequently, the specimen is cooled down and the test is repeated with the same conditions (test case B). Figure 7 compares the slippage load of different cycles of these two test cases. As it can be seen, the slippage loads in corresponding cycles of test cases A and B are almost equal. Furthermore, the slippage load of the very first cycle of loading is almost equal in all test cases. This means that, when the specimen is cooled down, the initial slippage load is recovered. 


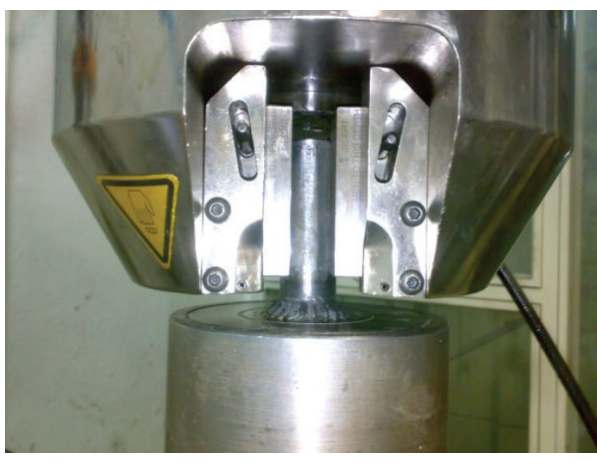

(a)

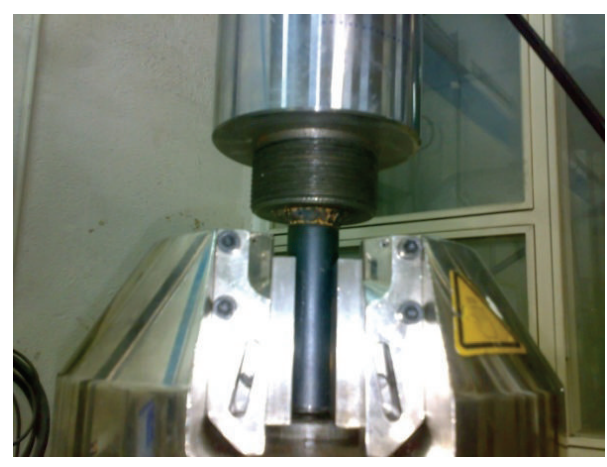

(b)

FIGURE 3: Connection of specimen to the actuator: (a) cylinder; (b) solid shaft.

TABLE 2: Slippage load for each cycle of loading.

\begin{tabular}{|c|c|c|c|c|c|c|}
\hline \multirow[b]{2}{*}{ Cycle number } & \multicolumn{2}{|c|}{ Case A } & \multicolumn{2}{|c|}{ Case B } & \multicolumn{2}{|c|}{ Case C } \\
\hline & $\begin{array}{l}\text { Slippage load } \\
(\mathrm{kN})\end{array}$ & $\begin{array}{l}\text { Difference from } \\
\text { average }(\%)\end{array}$ & $\begin{array}{l}\text { Slippage load } \\
(\mathrm{kN})\end{array}$ & $\begin{array}{l}\text { Difference from } \\
\text { average }(\%)\end{array}$ & $\begin{array}{l}\text { Slippage load } \\
(\mathrm{kN})\end{array}$ & $\begin{array}{c}\text { Difference from } \\
\text { average (\%) }\end{array}$ \\
\hline 1 & 219.41 & 14.82 & 216.16 & 15.66 & 212.68 & 14.06 \\
\hline 2 & 197.94 & 3.581 & 194.19 & 3.911 & 208.82 & 11.99 \\
\hline 3 & 190.85 & 0.129 & 189.95 & 1.643 & 201.61 & 8.124 \\
\hline 4 & 186.64 & 2.332 & 186.01 & 0.466 & 196.02 & 5.127 \\
\hline 5 & 184.30 & 3.556 & 178.72 & 4.366 & 190.93 & 2.397 \\
\hline 6 & 180.22 & 5.691 & 172.13 & 7.892 & 184.41 & 1.1 \\
\hline 7 & 178.31 & 6.690 & 171.00 & 8.497 & 176.67 & 5.251 \\
\hline 8 & - & - & - & & 170.34 & 8.646 \\
\hline 9 & - & - & - & & 164.87 & 11.58 \\
\hline 10 & - & - & - & & 158.26 & 15.12 \\
\hline Average & 191.1 & & 186.88 & & 186.46 & \\
\hline
\end{tabular}

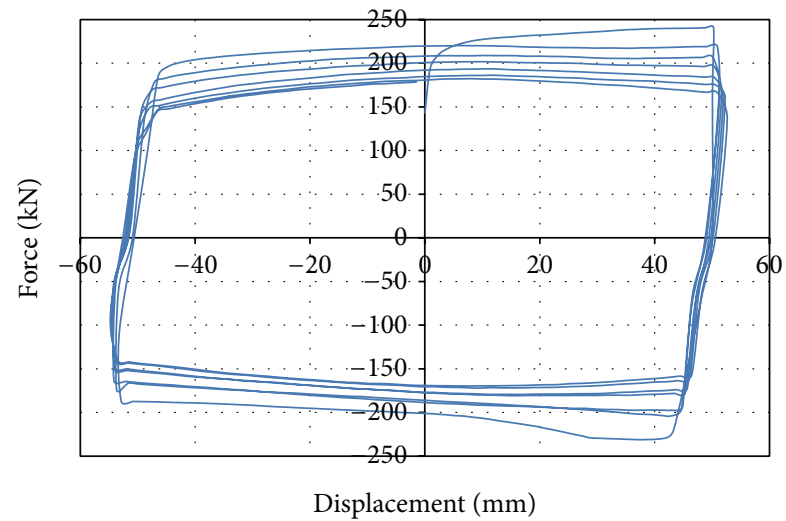

FIGURE 4: Experimental hysteretic force-displacement curve of the original CFD (case A).

\section{Analytical Study on the Effect of Wear}

As it was expected the slippage load, $F_{s}$, varies as the sliding length, $s$, tends to accumulate; that is,

$$
F_{s}(s)=\mu(s) N(s)=\mu(s) P(s) A,
$$

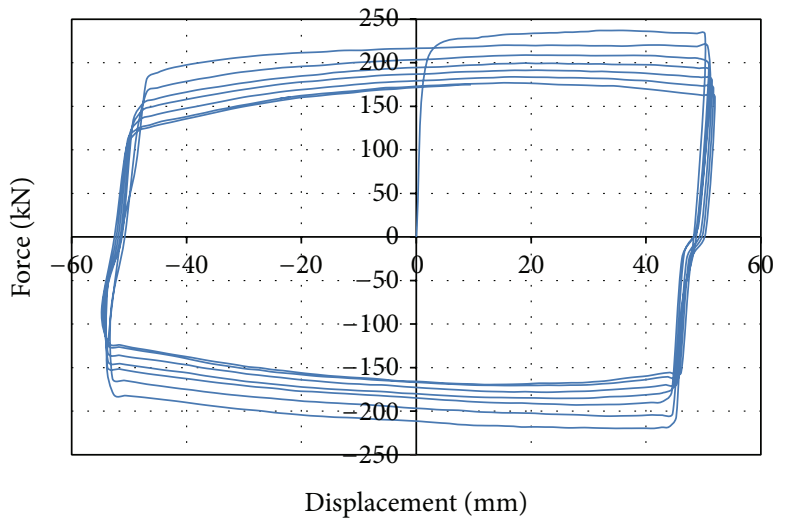

FIGURE 5: Experimental hysteretic force-displacement curve of the original CFD (case B).

where $A$ is contact area, $\mu$ is coefficient of friction, and $P$ is the contact pressure. As it was previously mentioned a number of strongly interacting structural and thermal processes contribute to dampers response. For the sake of simplicity let us just consider the effects of wear. A simple 


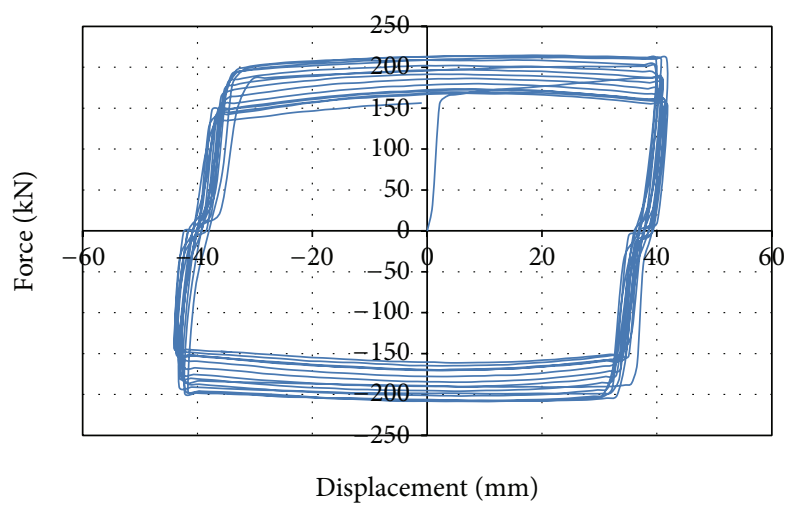

Figure 6: Experimental hysteretic force-displacement curve of the original CFD (case C).

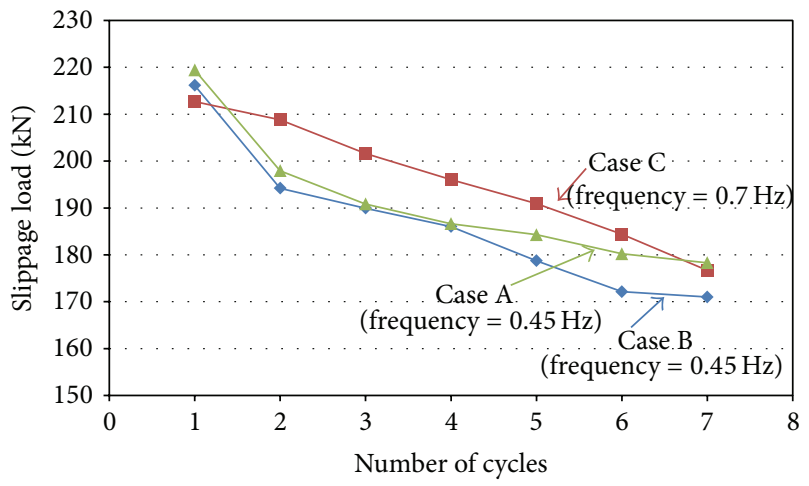

FIgURE 7: Slippage load versus number of cycles.

model used to describe sliding wear due to moving parts is the following Archard wear equation [12]:

$$
h=\kappa P s,
$$

where $h$ is the wear displacement, $\kappa$ is the specific wear coefficient, $s$ is the slippage length, and $P$ is the contact pressure. When $P$ may vary, the Archard equation is written as follows:

$$
d h=\kappa P(s) d s .
$$

For CFD, $P$ is calculated as follows [9]:

$$
P(s)=\frac{E \cdot \delta(s) \cdot\left(r_{o}^{2}-r_{i}^{2}\right)}{4 \cdot r_{i} \cdot r_{o}^{2}},
$$

where $r_{i}$ and $r_{o}$ are the inside and outside radii of the cylinder, respectively, and $\delta$ is the difference in the diameters of the shaft and the cylinder along the contact length. In the event of slippage, the shaft and external cylinder are possibly worn and their radii are reduced by $d h$. Therefore, the reduction of $\delta$ is equal to $4 \mathrm{dh}$. Consequently the contact pressure is reduced as follows:

$$
\Delta P(s)=\frac{E \cdot \delta(s) \cdot\left(r_{o}^{2}-r_{i}^{2}\right)}{4 \cdot r_{i} \cdot r_{o}^{2}}-\frac{E \cdot(\delta(s)-4 d h) \cdot\left(r_{o}^{2}-r_{i}^{2}\right)}{4 \cdot r_{i} \cdot r_{o}^{2}} .
$$

Substituting (3) into (5) leads to

$$
d(\Delta P(s))=\frac{\kappa \cdot E \cdot P(s) \cdot\left(r_{o}^{2}-r_{i}^{2}\right)}{r_{i} \cdot r_{o}^{2}} d s .
$$

The entire reduction of the contact pressure due to wear can be obtained by integrating over the interval which includes the sliding length:

$$
\Delta P(s)=\frac{\kappa \cdot E \cdot\left(r_{o}^{2}-r_{i}^{2}\right)}{r_{i} \cdot r_{o}^{2}} \int P(s) d s .
$$

Substituting (7) into (1) leads to

$$
\Delta F_{s}(s)=\frac{\kappa \cdot E \cdot\left(r_{o}^{2}-r_{i}^{2}\right) \cdot A}{r_{i} \cdot r_{o}^{2}} \int \mu(s) P(s) d s .
$$

It may be assumed that the contact pressure is constant during a cycle of loading. This assumption is on the conservative side since it makes the reduction of slippage load overestimated. Also $\mu$ is assumed to be constant for all cycles. The latter assumption is true, if mild wear occurs. If severe wear occurs, the coefficient of friction increases which in turn may lead to increase of the slippage load over the cycles. Several experimental tests are performed for CFD to find the limiting contact pressure which leads to severe wear and the contact pressure of the final prototype was selected to be less than the above limiting value. If sufficient experimental data is not available, the wear mechanism maps by Lim and Ashby [11] can be used. These assumptions lead to the following equation by removal of the constant values from integration signs of (7) and (8):

$$
\begin{gathered}
\Delta F_{s}=\alpha_{d} F_{s}, \\
\alpha_{d}=\frac{\kappa \cdot E \cdot s_{c} \cdot\left(r_{o}^{2}-r_{i}^{2}\right)}{r_{i} \cdot r_{o}^{2}}
\end{gathered}
$$

where $\Delta F_{s}$ is the reduction of the slippage load over a cycle of loading and $s_{c}$ is the total sliding length in that cycle. $\alpha_{d}$ is a positive dimensionless parameter which is developed by the authors and is referred to as coefficient of decay of slippage load.

The slippage load after the $n$th cycle of loading is called $F_{s n}$ and can be calculated as follows:

$$
F_{s n}=\left(1-\alpha_{d}\right)^{n-1} F_{s i},
$$

where $F_{s i}$ is the initial slippage force. Based on the requirements for displacement-dependent devices of ASCE/SEI 4106 [10], each device shall be loaded with 20 fully reversed cycles. The slippage force of each cycle must not differ by more than plus or minus $15 \%$ from the average value of slippage load. The average slippage load of 20 cycles of loading, $F_{s a}$, based on newly developed coefficient $\alpha_{d}$ can be calculated as follows:

$$
F_{s a}=\frac{F_{s i}}{20} \sum_{n=1}^{20}\left(1-\alpha_{d}\right)^{n-1}=\frac{F_{s i}}{20}\left[\frac{1-\left(1-\alpha_{d}\right)^{20}}{\alpha_{d}}\right] .
$$




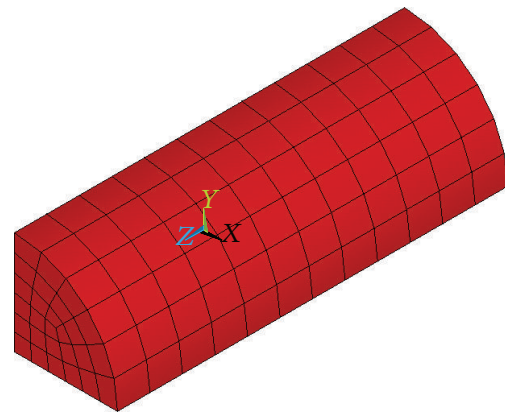

(a)

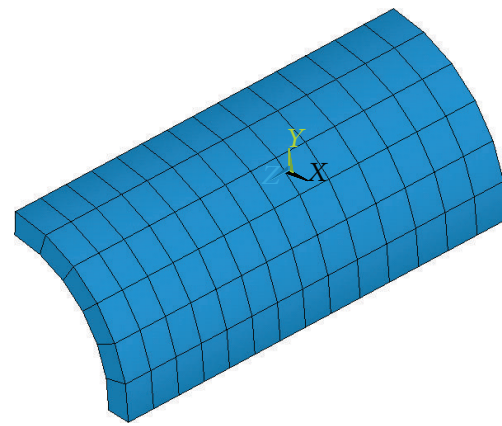

(b)

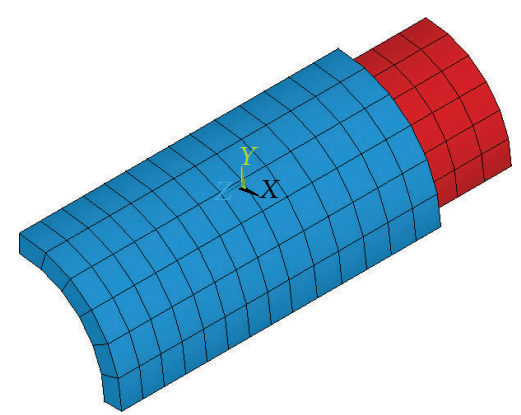

(c)

FIgURE 8: Numerical model of the original CFD; (a) shaft, (b) cylinder, and (c) assembled CFD.

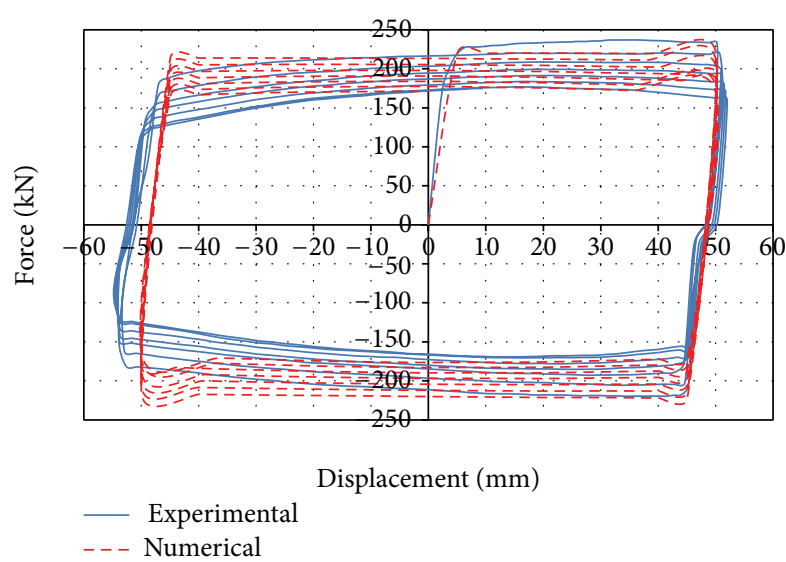

FIGURE 9: Comparison between numerical and experimental results (case B).

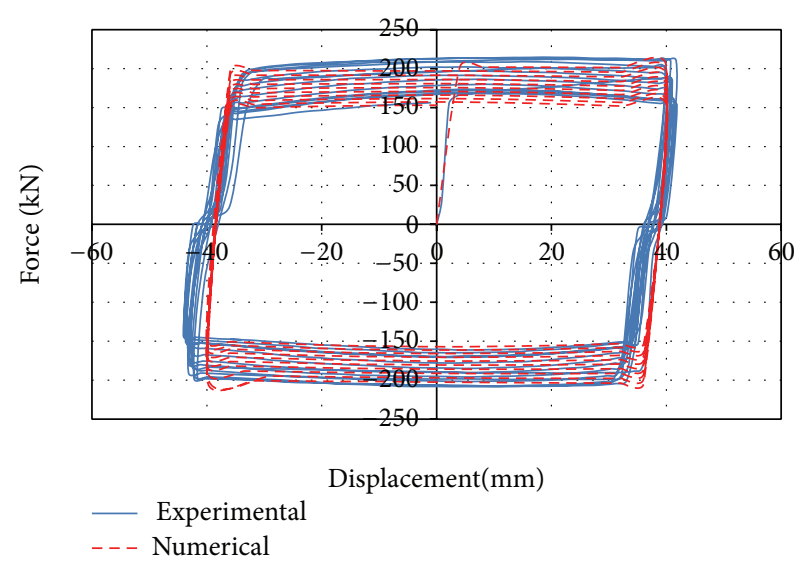

Figure 10: Comparison between numerical and experimental results (case $\mathrm{C}$ ).

The maximum deviation from average slippage load is calculated as follows:

$$
\frac{F_{s i}-F_{s a}}{F_{s a}}=\frac{20 \alpha_{d}-1-\left(1-\alpha_{d}\right)^{20}}{1-\left(1-\alpha_{d}\right)^{20}} .
$$

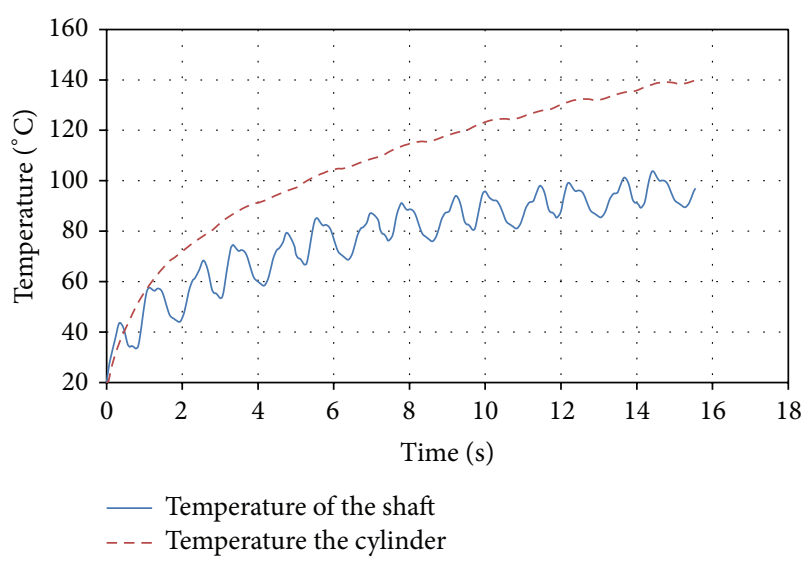

Figure 11: Comparison between temperatures of the shaft and cylinder for original CFD (case B).

Consequently, the critical value for $\alpha_{d}$, that is, the value which leads to $15 \%$ deviations from average, is $\alpha_{\text {cr }}=0.015$. The specific wear rate coefficient is determined by tests to be $\kappa=$ $1 \mathrm{e}-15 \mathrm{~m}^{3} / \mathrm{Nm}$ for CFD specified contact surfaces. Assuming that $s_{c}=200 \mathrm{~mm}$, the value of $\alpha_{d}$ becomes equal to 0.000186 which is well below the critical value; thus the issue of wear is not a concern for the tested CFD. This analytical result is in close agreement with the test results.

\section{Numerical Study of the CFD Considering Thermal-Structural Interaction}

Both analytical and experimental studies conclude that reduction of slippage load may be attributed to thermal deformation rather than wear effects. In order to find the effect of geometric parameters on thermal deformation of CFDs and reach the optimum design dimensions based on heat equilibrium of the internal elements within the damper, numerical simulations of the system are constructed. Threedimensional finite element model of the CFD prototype is developed as shown in Figure 8. Brick solid elements are used to model the shaft and the cylinder. These elements have twenty nodes with thermal-structural coupling capability. Coupling is modeled with 2-way or direct coupling. Surface 


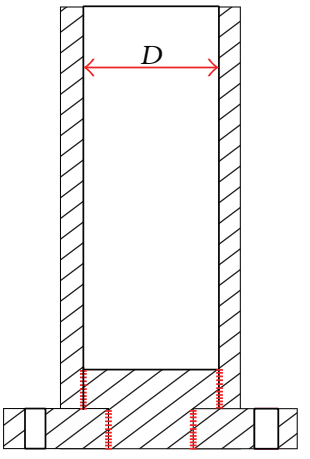

(a)

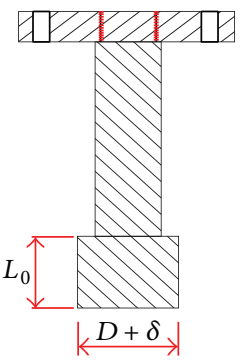

(b)

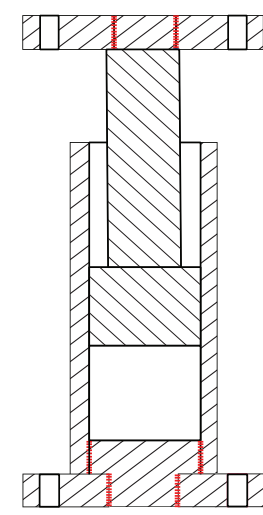

(c)

FIGURE 12: Improved CFD; (a) cylinder, (b) shaft, and (c) assembled CFD.

TABLE 3: Thermal characteristics of the numerical model.

\begin{tabular}{lcccc}
\hline Conductivity $\left(\mathrm{w} / \mathrm{m}^{2} \cdot \mathrm{k}\right)$ & Density $\left(\mathrm{kg} / \mathrm{m}^{3}\right)$ & Specific heat $(\mathrm{J} / \mathrm{kg} \cdot \mathrm{k})$ & Convection coefficient, $h\left(\mathrm{w} / \mathrm{m}^{2} \cdot \mathrm{k}\right)$ & Ambient temperature $\left({ }^{\circ} \mathrm{C}\right)$ \\
\hline 44 & 7850 & 460 & 60 & 20 \\
\hline
\end{tabular}

to surface contact is utilized to simulate the friction between the shaft and the cylinder at the contact length. Taking advantage of symmetry, only $1 / 4$ of the device is modeled. Convections are applied as surface loads on exterior surfaces of the cylinder and both sides of the shaft to account for heat loss into the surrounding environment. The rate of heat generation due to friction between contact surfaces is

$$
q=\gamma \cdot \tau \cdot v
$$

where $q$ is the heat flux, that is, thermal power per unit area, $\gamma$ is the fraction of frictional energy converted into heat, $\tau$ is the frictional stress, and $v$ is the sliding rate. Therefore, the heat absorbed by the solid shaft, $q s$, and the heat absorbed by the external cylinder, $q c$, are defined by

$$
\begin{aligned}
& q_{c}=\eta \cdot \gamma \cdot \tau \cdot v, \\
& q_{s}=(1-\eta) \cdot \gamma \cdot \tau \cdot v,
\end{aligned}
$$

where $\eta$ is the dimensionless coefficient of heat partitioning and is expressed by [13]

$$
\eta=\frac{1}{1+\sqrt{\left(\rho_{c} C_{c} K_{c}\right) /\left(\rho_{s} C_{s} K_{s}\right)}}
$$

where $\rho$ is the density, $C$ is the specific heat, $K$ is the thermal conductivity, and the subscripts, $c$ and $s$, represent the cylinder and shaft, respectively. Since both the cylinder and the shaft are made out of steel, coefficient of heat partitioning is calculated to be 0.5 which means that the heat absorbed by the solid shaft is equal to the heat absorbed by the external cylinder. In this study $\gamma$ is assumed to be equal to 1 . The modules of elasticity and Poisson's ratio are taken as $2.1 \mathrm{e} 5 \mathrm{MPa}$ and 0.3 , respectively. The ambient temperature is assumed to be $20^{\circ} \mathrm{C}$. Thermal characteristics of the model are given in Table 3. Cyclic displacements similar to experiments are applied to the model. The resulting hysteretic curves are obtained. For comparison between experimental and numerical results the hysteretic curves are superimposed on the same graph in Figures 9 and 10. As it can be seen, the numerical results closely match those obtained from experiments which in turn validate the numerical model.

\section{Improving CFD Geometry Utilizing Verified Numerical Models}

Both the cylinder and the shaft are made out of steel; thus the heat absorbed by the external cylinder is the same as the solid shaft. However, the solid shaft is much more massive than the cylinder and its temperature is less increased in comparison with the cylinder. This may overcome the convectional heat transfer which is significantly more for the cylinder rather than the shaft. This situation does not satisfy heat equilibrium and eventually leads to decay of slippage load. Figure 11 shows the comparison of the temperature time histories of two nodes, one at the outer face of the shaft and the other at the inner face of the cylinder, obtained from numerical analysis. This difference in temperature causes the decay of slippage load in consecutive cycles. To overcome the problem which affects the damper's performance, the geometry of the CFD is modified as shown in Figure 12 in order to satisfy favorable thermal equilibrium. In the improved geometry the radius of the shaft is slightly greater than the inner radius of the cylinder, resulting in a balance of mass distribution between the solid shaft and the cylinder. Furthermore, the length by which the heat is absorbed by the cylinder is increased. Figures 13 and 14 show the simulation for hysteretic behavior of an improved CFD which is redesigned for slippage load of $200 \mathrm{kN}$. Figure 13 is obtained for the same conditions of the experimental test cases $\mathrm{A}$ and $\mathrm{B}$, that is, frequency of $0.45 \mathrm{~Hz}$ and 7 cycles of loading, and Figure 14 is obtained for the same conditions of the experimental test case $\mathrm{C}$, that 


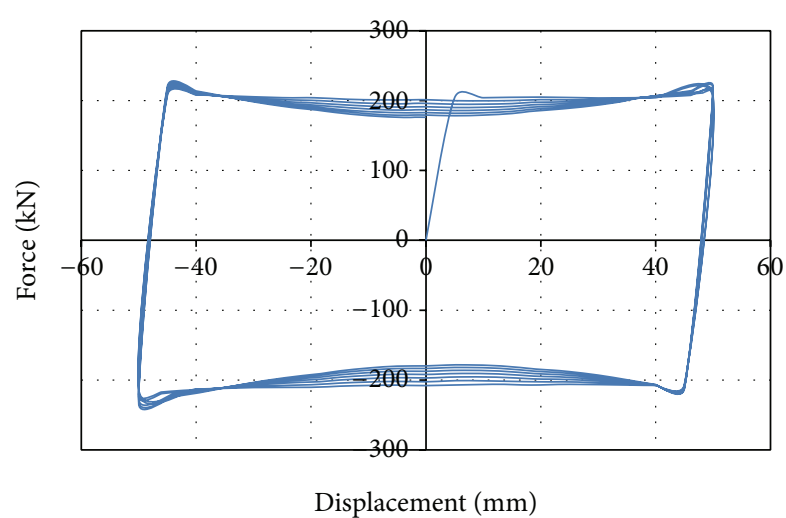

FIGURE 13: Hysteretic force-displacement curve of the improved CFD (7 cycles and frequency $=0.45 \mathrm{~Hz}$ ).

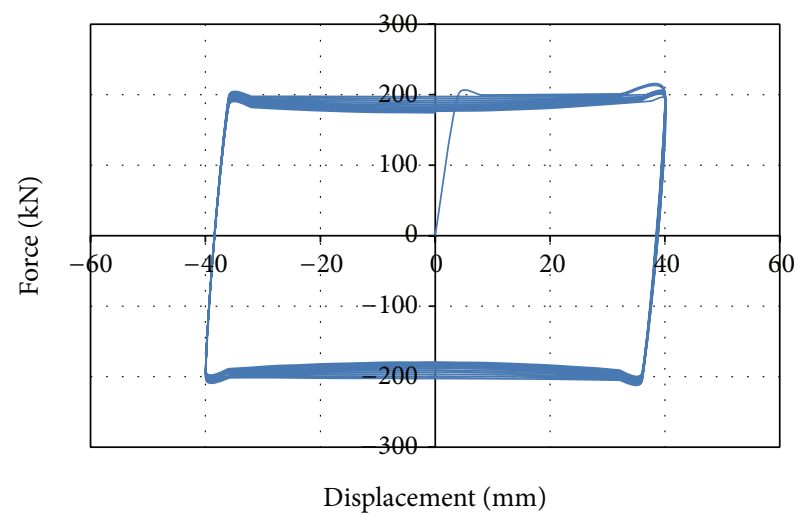

FIGURE 14: Hysteretic force-displacement curve of the improved CFD $(10$ cycles and frequency $=0.7 \mathrm{~Hz})$.

is, frequency of $0.7 \mathrm{~Hz}$ and 10 cycles of loading. As it can be seen, the stability of the hysteretic force-displacement curve is significantly increased. Figure 15 shows the comparison of the temperature time histories of two nodes, one at the outer face of the shaft and the other at the inner face of the cylinder. As it can be seen, there is a little difference between the nodal temperature time histories of the shaft and the cylinder which ultimately eliminates the drift of the slippage load.

\section{Conclusions}

The effect of dynamic loading on hysteretic behavior of cylindrical frictional dampers (CFDs) was investigated both experimentally and numerically. Experiments were carried out at two frequencies of $0.45 \mathrm{~Hz}$ and $0.7 \mathrm{~Hz}$. Results showed that the slippage load is drifted down in consecutive cycles of loading. It was also shown that this drift is mostly attributed to thermal deformation due to the accumulated heat generated by frictional forces. The hysteretic behavior of CFD was also investigated by numerical models considering direct thermal-structural coupling. These numerical models were verified by the hysteretic behavior of CFD obtained by the experiments. The verified numerical models were used to improve the geometry of the CFDs in order to

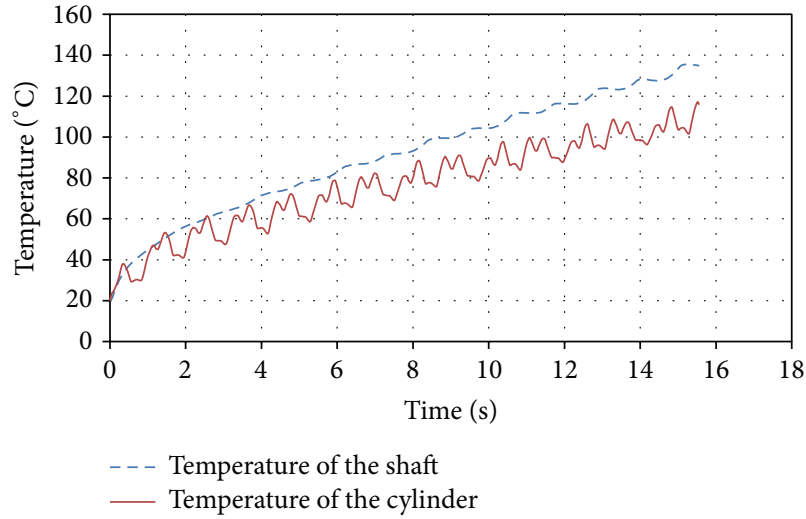

FIGURE 15: Comparison between temperatures of the shaft and cylinder for improved CFD $(7$ cycles and frequency $=0.45 \mathrm{~Hz})$.

create more thermal equilibrium. The improved geometry was incorporated into the numerical models which resulted in almost no degradation of the slippage load and stable hysteretic loops. A dimensionless parameter called coefficient of decay of slippage load was introduced by the authors which shows the effect of wear on the response of CFD. It was further shown that the problems which may arise from the wear are eliminated.

\section{Conflict of Interests}

The authors declare that there is no conflict of interests regarding the publication of this paper.

\section{References}

[1] A. S. Pall and C. Marsh, "Response of friction damped braced frames," ASCE Journal of Structural Division, vol. 108, no. 6, pp. 1313-1323, 1982.

[2] B. Wu, J. Zhang, M. S. Williams, and J. Ou, "Hysteretic behavior of improved Pall-typed frictional dampers," Engineering Structures, vol. 27, no. 8, pp. 1258-1267, 2005.

[3] I. D. Aiken and J. M. Kelly, "Earthquake simulator testing and analytical studies of two energy-absorbing systems for multistory structures," Earthquake Engineering Research Center, University of California UCB/EERC-90/03, Berkeley, Calif, USA, 1990.

[4] D. K. Nims, J. A. Inaudi, P. J. Richter, and J. M. Kelly, "Application of the energy dissipating restraint to buildings," in Proceedings of the ATC-17-1 Seminaron Seismic Isolation, Passive Energy Dissipation, and Active Control, pp. 627-638, Advanced Technology Council, March 1993.

[5] I. H. Mualla and B. Belev, "Performance of steel frames with a new friction damper device under earthquake excitation," Engineering Structures, vol. 24, no. 3, pp. 365-371, 2002.

[6] H. S. Monir and K. Zeynali, "A modified friction damper for diagonal bracing of structures," Journal of Constructional Steel Research, vol. 87, pp. 17-30, 2013.

[7] G. Chen, G. T. Garrett, C. Chen, and F. Y. Cheng, "Piezoelectric friction dampers for earthquake mitigation of buildings: design, fabrication, and characterization," Structural Engineering and Mechanics, vol. 17, no. 3-4, pp. 539-556, 2004. 
[8] A. Agrawal and J. Yang, "A semi-active electromagnetic friction damper for response control of structures," in Advanced Technology in Structural Engineering, pp. 1-8, ASCE, Philadelphia, $\mathrm{Pa}, \mathrm{USA}, 2000$

[9] M. Mirtaheri, A. P. Zandi, S. S. Samadi, and H. Rahmani Samani, "Numerical and experimental study of hysteretic behavior of cylindrical friction dampers," Engineering Structures, vol. 33, no. 12, pp. 3647-3656, 2011.

[10] "Seismic rehabilitation of existing buildings," Tech. Rep. ASCE/SEI 41-06, American Society of Civil Engineers, Reston, Va, USA, 2006.

[11] S. C. Lim and M. F. Ashby, "Wear-mechanism maps," Acta Metallurgica, vol. 35, no. 1, pp. 1-24, 1987.

[12] J. F. Archard, "Wear theory and mechanisms," in Wear Control Handbook, M. B. Peterson and W. O. Winer, Eds., ASME, New York, NY, USA, 1980.

[13] H. Blok, "Fundamental mechanical aspects in boundary lubrication," SAE Transactions, vol. 46, pp. 54-68, 1940. 

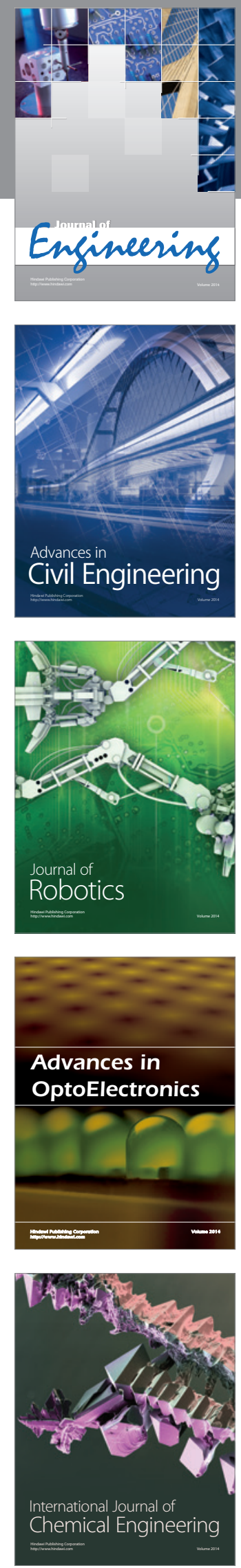

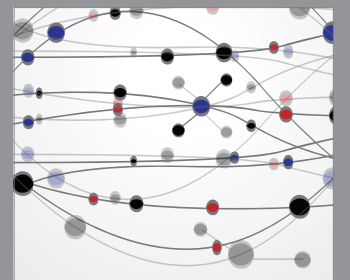

The Scientific World Journal
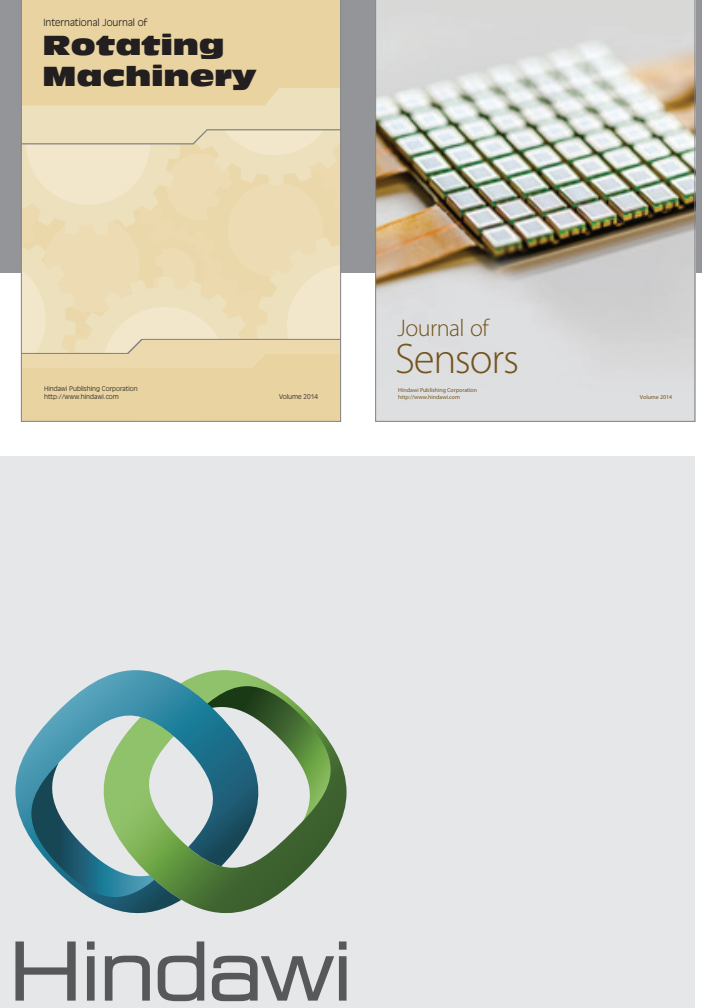

Submit your manuscripts at http://www.hindawi.com
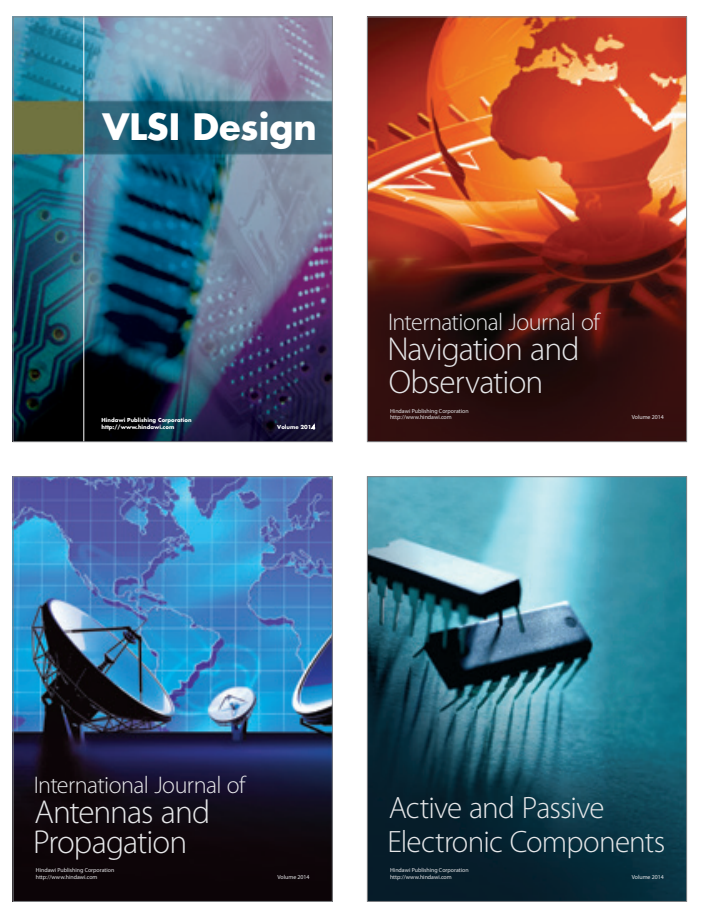
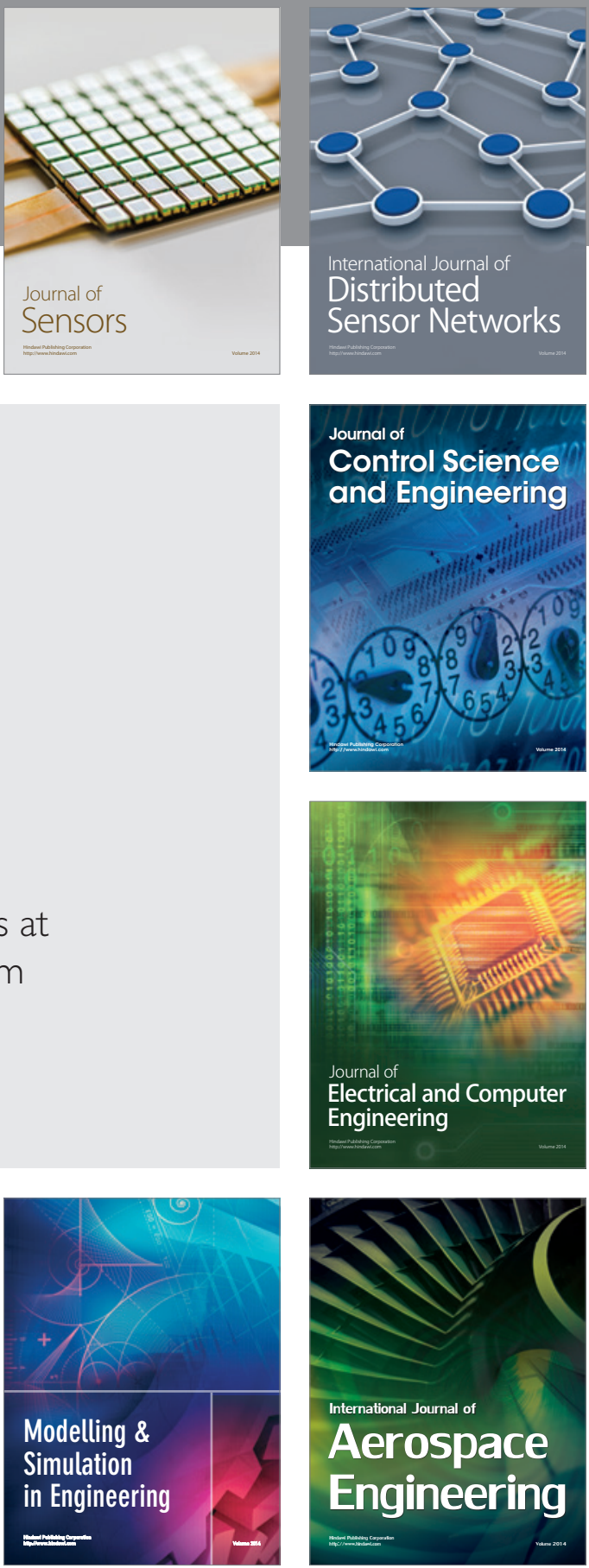

Journal of

Control Science

and Engineering
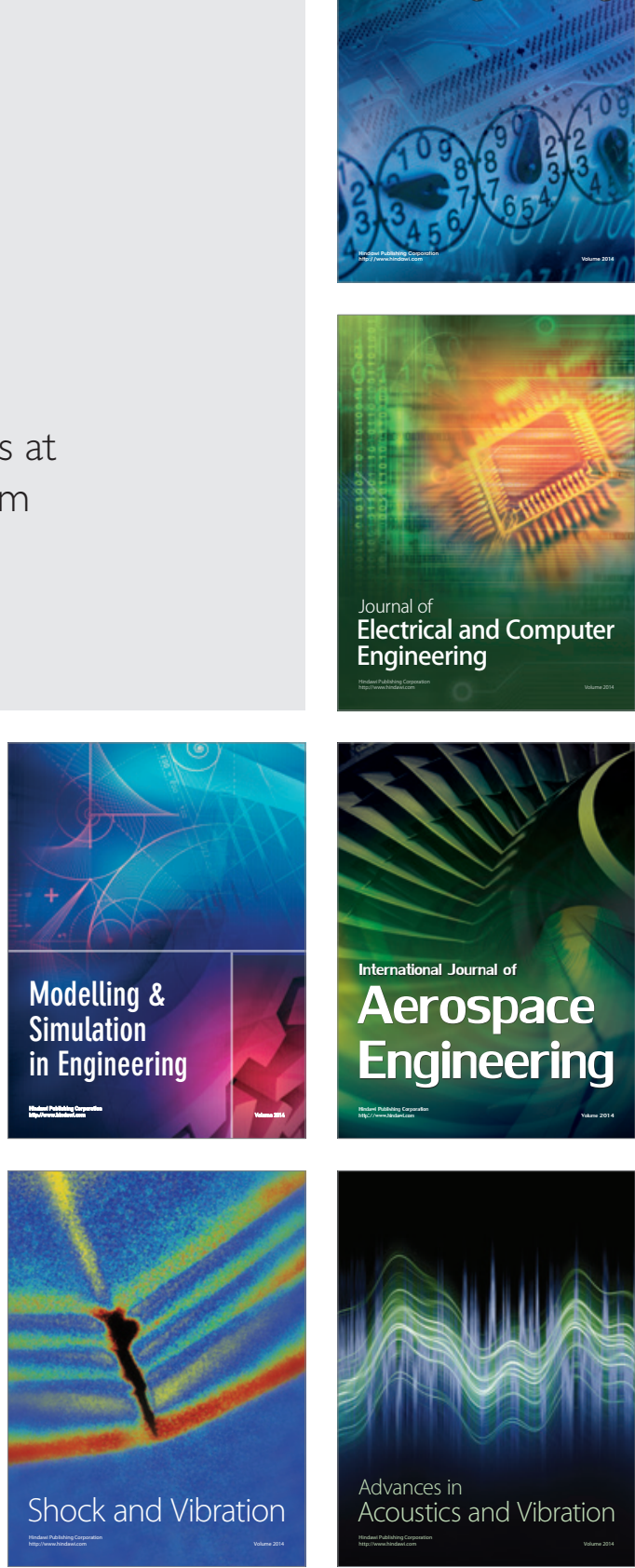